\title{
HYPOTHETICAL FEASIBILITY OF USING STRESS BIOMETRICS IN STUDENTS WITH CORTICAL VISUAL IMPAIRMENT
}

\author{
Victor M. Pedro $^{1}$, Juby Mathew ${ }^{1}$ and Elena Oggero ${ }^{2,3}$ \\ ${ }^{1}$ iBrain - International Institute for the Brain, New York, NY \\ ${ }^{2}$ Electrical and Computer Engineering Department, University of Wyoming, Laramie, WY \\ ${ }^{3}$ Vestibular Technologies, LLC, Cheyenne, WY
}

Corresponding Author: Victor M. Pedro

International Institute for the Brain - iBRAINnyc.org - 311 East 94th St. New York, NY 10128, USA

Tel: +1 646-315-1548

Email: vpedro@iBRAINnyc.org

https://doi.org/10.34107/BiomedSciInstrum.57.04184

\begin{abstract}
Cortical Visual Impairment (CVI) is resultant from neurological injury and damage to visual pathways or vision centers in the brain. CVI is sometimes undiagnosed in individuals with brain injuries due to the complexity of the human visual system. The International Institute for the Brain (iBrain) is a specialized school for students ages 5 to 21 who have a brain disorder or an acquired brain injury. They often present with noticeable CVI. While there are various metrics and interventions for the pediatric population, the adequacy has been lacking in terms of the vulnerability of this non-verbal population. Assessing the safety and effectiveness of rehabilitative interventions for this fragile student population can be challenging as most traditional metrics cannot be used. In this methodological review paper, available metrics were investigated and their applicability for this specific population is discussed with the end goal of identifying the best metrics that could be used to determine treatment effectiveness and providing a way for monitoring adverse effects. Combining pulse oximetry, cortisol response sensor, and galvanic skin response as biometrics theoretically offers a comprehensive assessment of autonomic activity and responses and establishes objective measures to identify treatment outcomes and adverse reactions. However, future experimental studies are needed to verify if the proposed protocol is feasible and if it is well tolerated by the iBrain students before it can be implemented to monitor adverse reaction to intervention and as a potential treatment outcome measure for children affected by CVI.
\end{abstract}

Keywords: Practice-based clinical research, Cortical visual impairment, Galvanic skin response, Pulse oximetry and heart rate, Cortisol dermal response sensors, Wearable sensorU+0073fi

\section{INTRODUCTION}

Cortical Visual Impairment (CVI) is a decreased visual response due to a neurological problem affecting the brain's visual system [1-2]. This impairment is due to damage to the posterior visual pathways, resulting in characteristic observable behaviors depending upon which of the visual centers or association area is involved [2]. About 30-40\% of visually-impaired children have cortical visual impairment with an expansive range of visual dysfunction including severe blindness [3]. It can be improved over time if early interventions are provided for the student, decreasing the possibility of blindness [1-2]. Early interventions include assistive technology, hearing services, speech and language services, counseling and training for a family, medical services, occupational therapy, physical therapy and vision services [3]. Monitoring the impact of any intervention on such a fragile, often non-verbal population is essential, especially if they present with seizure activity.

At the International Institute for the Brain (iBrain), the student population ranges from the ages 5 through 21 years of age, all of which have sustained a brain injury and/or suffer a brain-based disorder. The students are nonverbal, non-ambulatory (wheelchair-dependent), and all require a full-time 1:1 